\title{
Force Adaptive Multi-spectral Imaging with an Articulated Robotic Endoscope
}

\author{
David P. Noonan ${ }^{1,2}$, Christopher J. Payne ${ }^{2,3}$, Jianzhong Shang ${ }^{2}$, Vincent Sauvage ${ }^{1,2}$, \\ Richard Newton $^{1,2}$, Daniel Elson ${ }^{1,2}$, Ara Darzi ${ }^{1}$, and Guang-Zhong Yang ${ }^{2,3}$ \\ ${ }^{1}$ Department of Surgery and Cancer, Imperial College London \\ ${ }^{2}$ Institute of Biomedical Engineering, Imperial College London \\ ${ }^{3}$ Department of Computing, Imperial College London \\ \{dnoonan, cjp04,j.shang, v.sauvage, r.newton\}@imperial.ac.uk \\ \{ds.elson, a.darzi,g.z.yang\} @imperial.ac.uk
}

\begin{abstract}
Recent developments in optical spectroscopic techniques have permitted in vivo, in situ cellular and molecular sensing and imaging to allow for real-time tissue characterization, functional assessment, and intraoperative guidance. The small area sensed by these probes, however, presents unique challenges when attempting to obtain useful tissue information in-vivo due to the need to maintain constant distance or contact with the target, and tissue deformation. In practice, the effective area can be increased by translating the tip of the probe over the tissue surface and generating functional maps of the underlying tissue response. However, achieving such controlled motions under manual guidance is very difficult, particularly since the probe is typically passed down the instrument channel of a flexible endoscope. This paper describes a force adaptive multi-spectral imaging system integrated with an articulated robotic endoscope that allows a constant contact force to be maintained between the probe and the tissue as the robot tip is actuated across complex tissue profiles. Detailed phantom and ex-vivo tissue validation is provided.
\end{abstract}

Keywords: fluorescence spectroscopy, articulated robot, force control, in vivo tissue characterization.

\section{Introduction}

Point based spectroscopic approaches such as diffuse reflectance, Raman and fluorescence spectroscopy have been shown to be clinically useful for in vivo assessment of cancer, atherosclerosis and ischemia. Current techniques under development include visible diffuse light spectroscopy applied in the colon to detect colonic ischemia [1], the use of fluorescence spectroscopy to detect atherosclerotic lesions [2] and the use of Raman sensing to detect cervical cancer [3]. There has been increasing focus in recent years on simultaneous large area surveillance with high spectral resolution [4], (and therefore high sensitivity and specificity), potentially with multiple labels and multimodal spectroscopy. Simultaneously the continued development of targeted fluorescence probes that provide a specific spectral profile offer a further example of 
the importance of being able to trace a path across the tissue surface in order to investigate the localization of the marker whilst still achieving a good detection of the marker fluorescence on top of the optical background signal.

In practice, due to the small sensing area of these techniques and the common requirement of tissue contact, performing large area surveillance and repeated examination is difficult due to in vivo tissue deformation and the difficulty in manually maintaining the probe-tissue distance. Previous results have shown that these techniques are affected by the pressure exerted between the probe and the tissue surface [5] due to the following effects: (a) the coupling efficiency between the fibres and the tissue, which depends on the refractive index mismatch between the two; (b) the change in the optical scattering properties of the tissue due to compression; and (c) the increase in signal from deeper tissue layers with increasing pressure due to the displacement of the upper layers, (which can be a particularly important consideration when sensing layered tissues such as epithelial structures).

The purpose of this paper is to demonstrate a force adaptive multi-spectral imaging system integrated with an articulated robotic endoscope. This allows a constant contact force to be maintained between the probe and the tissue as the robot tip is translated across complex tissue profiles. A similar approach for measuring contact forces remotely has been employed for both fixed transformations between the sensor and contact site [6] [7] and flexible transformations [8]. An additional technical advantage of the system described in this paper is that the estimation of distal tip position allows for localization of specific spectral responses within the field of view. Detailed validation is provided using a multi-spectral phantom as well as results demonstrating successful force adaptive acquisition of tissue autofluorescence on ex-vivo liver tissue samples.

\section{Methods}

\subsection{Articulated Robotic Endoscope Design and Operation}

The articulated robotic endoscope used in this paper features five controllable degrees of freedom arranged into three serially connected link segments and attached to a $500 \mathrm{~mm}$ long rigid aluminum shaft. The degrees of freedom are arranged into two universal joints (intersecting pitch and yaw) at the distal and medial joints and a single yaw DoF at the proximal joint. Each DoF is independently addressable and capable of actuating $\pm 45^{\circ}$, enabling the device to servo its tip over a large area $\left( \pm 90^{\circ}\right.$ vertically and $\pm 135^{\circ}$ horizontally). An advantage of the serially connected joint architecture is that the proximal/medial joint(s) can be used to avoid obstacles, such as anatomical structures or other instrumentation, to safely position the distal joint at a more advantageous position. As can be seen in Fig. 1(a), the distal joint alone can then continue scanning to provide the operator with both visual feedback and the ability to interact at the target site.

The diameter of the shaft and articulated segments is $12.5 \mathrm{~mm}$ so as to be compatible with existing trocar ports. Each DoF is actuated by an embedded $4 \mathrm{~mm}$ diameter Brushless DC micromotor (Namiki Precision Jewel Ltd, SBL04-0829) with a 337:1 planetary gearbox. The rotary motion of the motor is converted to joint rotation via an 
internal mechanism (patent pending). Joint position is estimated by counting a single pulse output from the motor drive electronics for each full rotation of the motor. The length of the proximal and medial segments is $44 \mathrm{~mm}$ while the distal segment is $36 \mathrm{~mm}$ long. The device also features three internal channels $(2 * 3 \mathrm{~mm}, 1 * 1.8 \mathrm{~mm})$. The latter houses power and signal lines for the motors while the two other channels can be used for various additional instrumentation. An IntroSpicio ${ }^{\mathrm{TM}} 115$ Micro CCD video camera (Medigus Ltd, Israel) and a custom fluorescent imaging probe, which will be further described in Section 2.2, are used for this study.

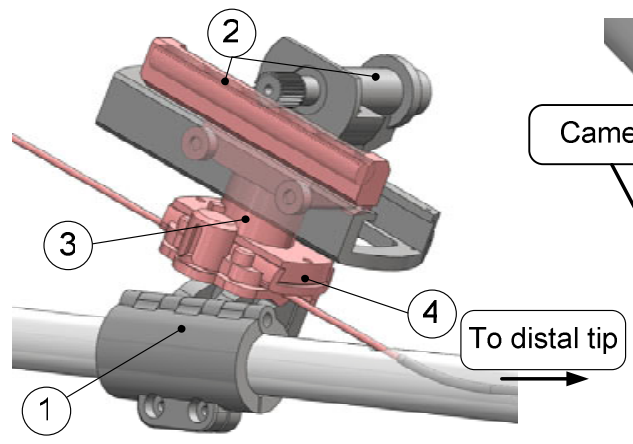

(a)

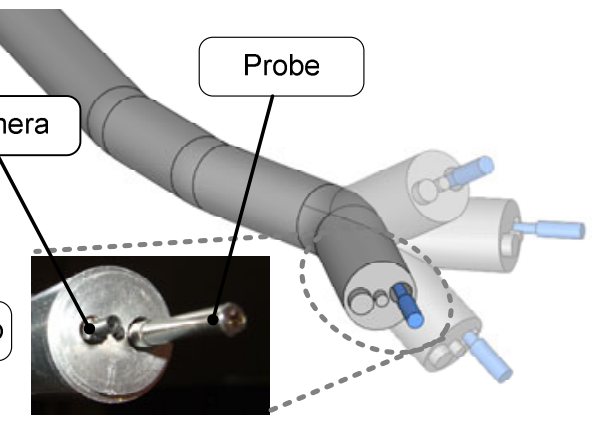

(b)

Fig. 1. (a) Schematic illustrating the force adaptive linear servoing mechanism. Moving parts are marked in red. It consists of four key components: a clamp (1) which attaches to the robot shaft, a Maxon RE10 Brushed DC motor driving a rack and pinion linear slider (2), a Nano17 F/T sensor (3) and a housing to clamp and thus translate the imaging probe (4) (b) A CAD schematic illustrating the concept of the robotic scanning device using controlled actuation of the distal tip. The $1.8 \mathrm{~mm}$ Medigus Camera and fluorescent imaging probe are also marked.

\subsection{Force Sensitive Probe Operation}

One key component of the articulated robotic device is a force sensitive linear servoing mechanism, into which the proximal end of the fluorescent imaging probe is clamped. The mechanism is located approximately $500 \mathrm{~mm}$ from the distal opening of the robot's $3 \mathrm{~mm}$ biopsy channel at the tip of the device. An embedded ATI Nano17 6axis Force/Torque sensor (SI-12-0.12) measures the axial load along the central axis of the imaging probe. Naturally, inaccuracies in the absolute force measurement will be introduced due to friction in the instrument channel, which will increase with the tortuousity of the robot (due to frictional interface effects along the length of the probe). However, the purpose of the system is not to provide the operator with an accurate absolute force measurement between the probe and the tissue, but rather to maintain a constant force during image acquisition. It is assumed that the force measured remotely from the tip and transmitted through the probe is sufficiently representative of the tip interaction force to drive the controller. Additionally, the probe is only expected to undergo a linear translation of less than $3-4 \mathrm{~mm}$ during operation which significantly reduces the negative effects of the long transmission distance. These issues will be further discussed in Section 3. 
Fig. 1(a) illustrates the F/T sensor clamped into the rack and pinion based linear servo mechanism. The pinion (24t, MOD 0.25$)$ is actuated via a brushed DC geared motor (Maxon RE10 6V, 16:1 gearhead, MR Type Integrated 256cpr Quadrature Encoder). The rack is seated in a groove which constrains all but a translational degree of freedom along the centerline of the imaging probe. A rack and pinion system was chosen for back-drivability, ease-of-use and so as to provide a fast response time. The probe is fed directly into the internal biopsy channel to prevent undesirable deflections or buckling as it is loaded axially.

A closed-loop force control scheme was created in the LabView development environment. The force along the centerline of the probe is measured by the force-torque sensor and acquired using a NI-6221 acquisition card at 500Hz. This signal is then fed to a Proportional-Integral controller and the output signal is processed by a differential operational amplifier (so as to allow bipolar control of the motor) which in turn forms the input of a pulse-width modulation-based speed controller for the DC motor (Maxon, LSC 30/2 linear 4Q Servoamplifier). The speed controller has an internal feedback loop which uses the integrated quadrature encoder to regulate the motor speed according to the input signal described above.

Fluorescence emission measurements were taken using a $2 \mathrm{~mm}$ diameter flexible fibre-optic probe (Romack, V.A., USA) consisting of 7 hexagonally assembled fibres, each having a $200 \mu \mathrm{m}$ core diameter and a 0.22 numerical aperture. Excitation light was provided by a $375 \mathrm{~nm}$ laser diode collimated with a $4.6 \mathrm{~mm}$ focal length lens and focused into a central excitation fibre with an $8 \mathrm{~mm}$ focal length lens. The fluorescence emission light collected by the six fibers surrounding the central fibre was arranged into a linear array which was imaged onto the $80 \mu \mathrm{m}$ wide slit of a spectrograph (Specim Ltd, Finland). A 405nm long-pass filter was inserted in to block the reflected excitation light and the light was dispersed by a prism-grating-prism element of the spectrograph and detected with a cooled CCD camera (Retiga EXI, QImaging, $1392 \times 1040$ pixels). The camera and the beam shutter were also controlled through LabView (exposure time phantom, 1ms; exposure time liver, 10ms; region of interest 1392x110) and the autofluorescence spectrum was acquired in a single shot with a frame-rate of $130 \mathrm{~ms}$. Image acquisition from the onboard Medigus camera is also synchronized with the autofluorescence signal acquisition, which allows the immediate localization of the probed site within the camera field of view.

\section{Experimental Design and Results}

Three experiments were performed in order to access the efficacy of system. The first was a system characterization to evaluate what contact force should be used, the second employed a fluorescent stained phantom to validate the ability of the system to accurately reconstruct a multi-spectral map and finally, the third experiment was performed on ex-vivo fowl liver to demonstrate the ability of the system to maintain sufficient contact with the tissue to acquire autofluorescence spectra. The first set of experiments were performed to characterize: a) the effect of different angular displacements of the distal tip on the minimum force required to advance the imaging probe; and b) the effect of different contact forces on the intensity of the resulting spectroscopy image. 
To characterize the effect of different angular displacements, the distal tip was servoed to five different positions (straight and locations in each of the four quadrants of the Cartesian co-ordinate system). At each position, the setpoint of the force controller was increased in increments of $10 \mathrm{mN}$ until the probe advanced and made contact with a fluorescein stained silicone phantom. The results show that larger forces are required to move the imaging probe when the tip is deflected than when it is straight ( $20 \mathrm{mN}$ straight, $30-40 \mathrm{mN}$ at deflections of $\pm 12^{\circ}$ on each axis of the universal joint) . This is due to friction interference effects between the imaging probe and the channel inside the robot as it deflects. All subsequent experiments were carried out with a minimum setpoint of $40 \mathrm{mN}$ and for all experiments described in this paper, only the distal tip of the robot was actuated.

Further experiments were performed to characterize the spectral response of the probe for different contact forces with ex-vivo fowl liver tissue (Fig. 2). The probe was advanced into contact with the tissue and the contact force was increased in $10 \mathrm{mN}$ increments up to a limit of $150 \mathrm{mN}$ as spectroscopic images were acquired. The results indicated that while the magnitude of the tissue autofluorescence remains constant for all contact forces, the effect of ambient light on the spectral trace decreases in proportion with increasing contact force. The presence of this signal is due to diffusion of the illumination light through the tissue to the detection fibers even though the probe is in full contact with the tissue. Based on feedback from the onboard camera, it can be seen that as the tissue undergoes higher deformation due to high contact forces, less ambient light is detected. However, these same high contact forces do not affect the intensity of the autofluorescence signal from the tissue. These results indicate that with this system we can maintain a low but constant force that achieves a sufficient optical signal without applying excessive load to the tissue.
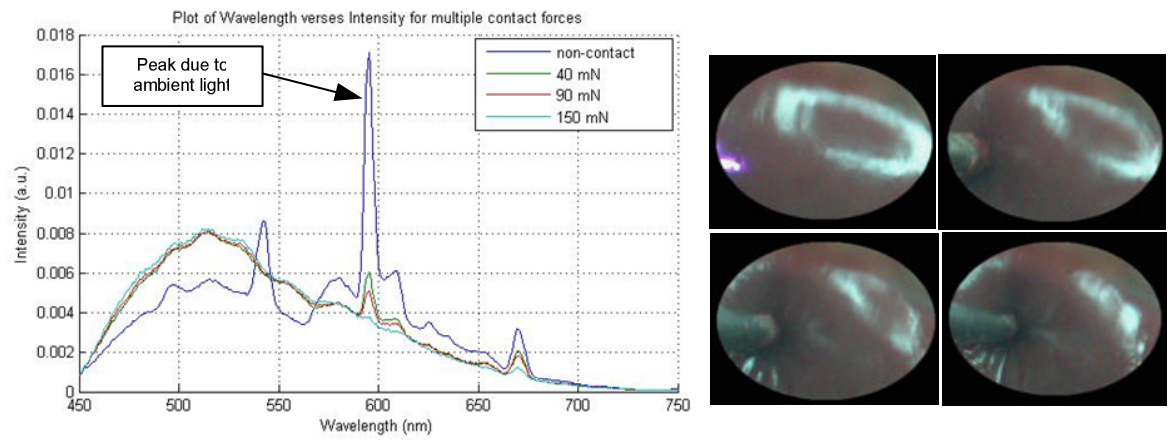

Fig. 2. Plot of Intensity versus Wavelength for contact forces of $0 \mathrm{mN}, 40 \mathrm{mN}, 90 \mathrm{mN}$ and $150 \mathrm{mN}$. The synchronized images from the onboard camera illustrate the probe-tissue interaction for the four contact forces (clockwise from top left) $0 \mathrm{mN}, 40 \mathrm{mN}, 90 \mathrm{mN}$ and $150 \mathrm{mN}$.

In order to validate the ability of the system to accurately trace a trajectory and reconstruct the underlying multi-spectral fluorescence images, a double stained phantom was developed. The silicone phantom featured four horizontal lines mounted on a flat surface. The first and third lines consisted of yellow fluorescent dye while the second and fourth lines consisted of green fluorescent dye. The lines were approximately $1.5 \mathrm{~mm}$ thick and were separated by a gap of approximately $1.5 \mathrm{~mm}$. 

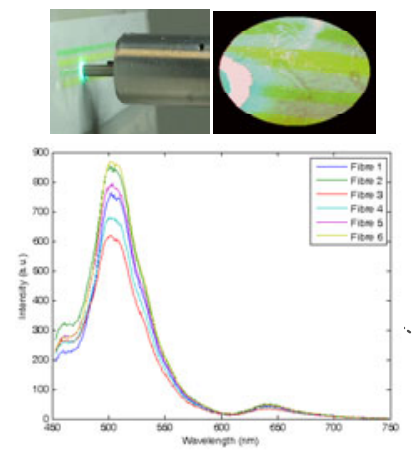
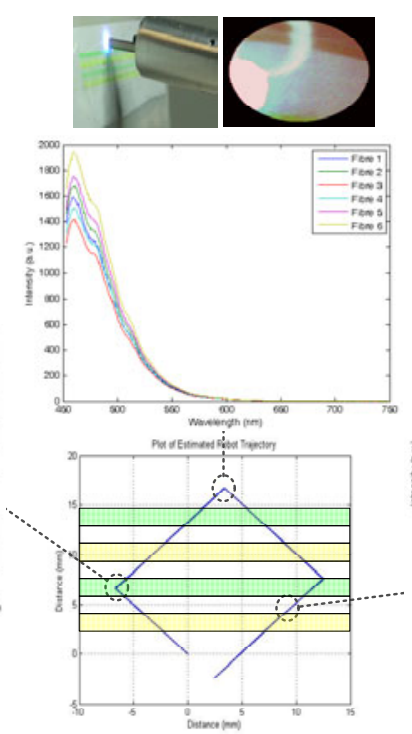
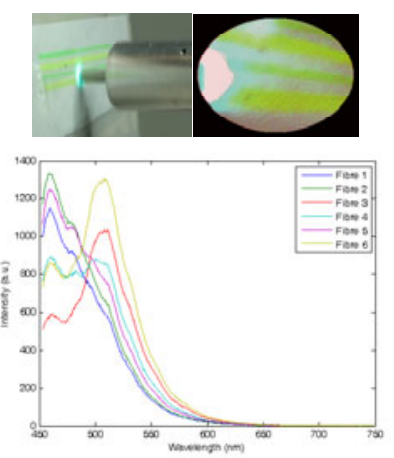

Fig. 3. Plot of the trajectory of the robot tip as it passes over the green and yellow fluorescence dye. Three results sequences displaying the spectral information (bottom) the onboard camera (top-right) and an external camera for visual confirmation of the spectral result (top-left) are also shown.

In order to obtain continuous multi-spectral measurements along 2D scan patterns, each of the separate components (robot control, linear actuator control, onboard camera acquisition and fluorescence image acquisition) were synchronized and the robot was driven under joystick control along a trajectory. This synchronization allows the spectral information at each acquisition to be localized along the tip trajectory using the estimation of tip position recorded from the motor pulses. The location of each image can be confirmed by the use of the onboard camera and a second, external camera which was used solely for validation purposes. Additionally, the cameras were used to confirm that the probe maintained contact with the surface at all times. Since the probe tip moves in an arc during robotic joint actuation the linear servoing mechanism must actuate the probe in order to maintain contact during all stages of the image acquisition. A similar situation would arise with biological tissue where the surface to be probed would have a different profile to the arc traced by the robot tip.

Fig. 3 shows the robot tip trajectory projected onto a 2D plane (located at the robot distal face when the robot is straight) and a sample of three result sets from three example points along the trajectory. Each result sequence shows the spectral image acquired at that location (bottom), the onboard camera view (top-right) and the external camera view (top-left). The location of the spectral peak at different locations (paper: $460 \mathrm{~nm}$, yellow dye: $515 \mathrm{~nm}$, green dye: $505 \mathrm{~nm}$ and $640 \mathrm{~nm}$ ) clearly illustrate the ability of the system to differentiate between the different wavelengths recorded at the different locations. The results on the right hand side of Fig. 3 are particularly interesting as different fibers within the imaging probe display different excitation wavelengths. During these acquisitions, the probe was straddling the boundary of 
more than one excitation. This indicates that higher resolution multi-spectral information can be obtained by incorporating the relevant spatial distribution of the fibers in the probe.

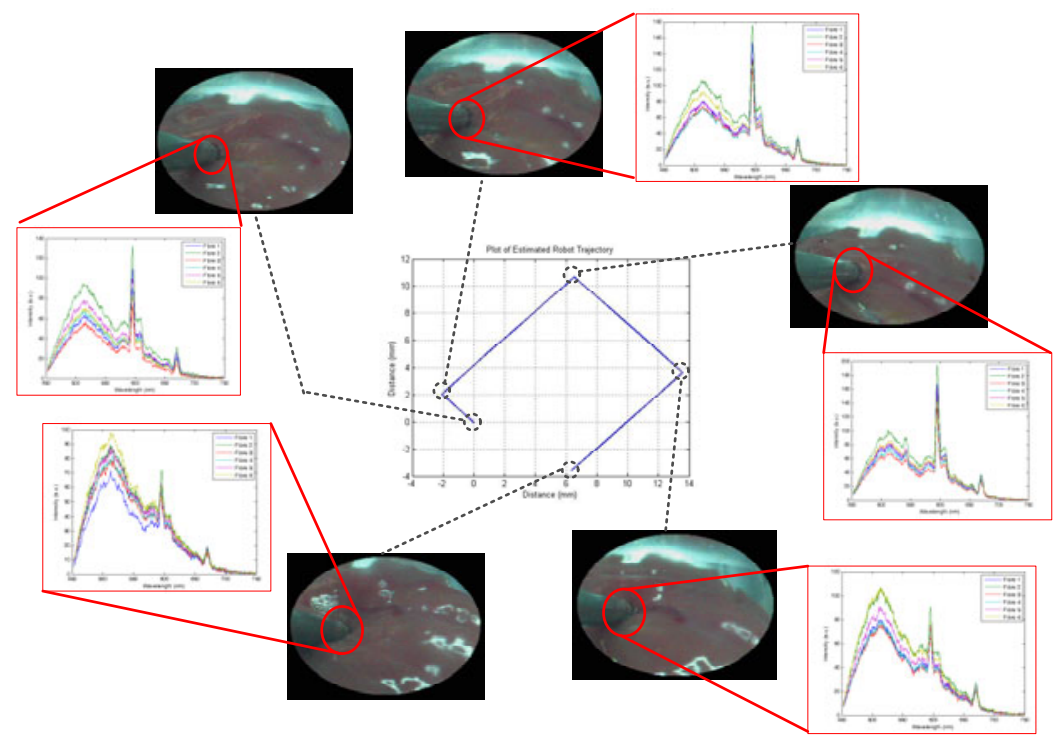

Fig. 4. (middle) 2D plot of the trajectory of the probe tip with five results sets indicated. Each results set displays the spectral information and the synchronized onboard camera image.

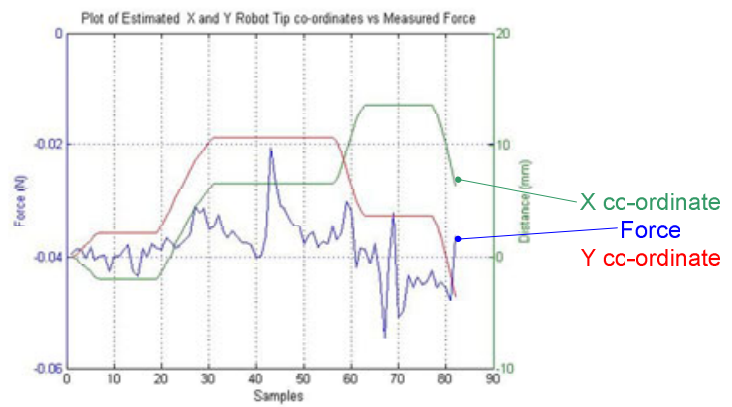

Fig. 5. Plot of the $X$ and $Y$ co-ordinates of the probe tip (green and red respectively) verses the force measured by the F/T sensor (blue)

To demonstrate the ability of the system to employ the protocol described above to record autofluorescence from biological soft tissue a similar experiment was performed on ex-vivo fowl liver. These results are shown in Fig. 4. Images from the onboard camera are highlighted as well as the spectral information captured by the probe. The magnitude of the autofluorescence peak in the spectra (peak approx. $510 \mathrm{~nm})$ remains constant for all acquisitions, indicating that good contact is maintained throughout the experiment. Fig. 5 illustrates the relationship between the 
movement of the tip and the measured force during this acquisition sequence. As can be seen from the plot the force controller maintains a constant contact force of $40 \mathrm{mN}$ for the duration of the run. Deviations from the constant $40 \mathrm{mN}$ force occur after approx. 43 and 66 samples. These deviations are due to changes in the tissue surface profile which cause the probe to almost lose contact with the tissue or become buried deeper into the tissue. In each of these cases the controller servos the probe to maintain the $40 \mathrm{mN}$ force. This motion can be observed in the onboard camera.

\section{Discussion and Conclusion}

The results presented in this paper demonstrate the ability of a robotically-assisted approach for consistent spectroscopic sensing by overcoming the challenges of manual probe manipulation. By decoupling the operator from the complex manipulations required to obtain such images, the focus of the clinical investigation can be shifted towards identifying potential target sites and interpreting the spectroscopic information. The ability of the proposed system to obtain continuous multi-spectral information along 2D scan patterns across complex surface profiles, while maintaining a constant contact force, has clear potential clinical applications in areas such as tumour margin identification and localization of fluorescence markers.

\section{References}

1. Friedland, S., Benaron, D., Coogan, S., Sze, D.Y., Soetikno, R.: Diagnosis of chronic mesenteric ischemia by visible light spectroscopy during endoscopy. Gastrointestinal Endoscopy 65, 294-300 (2007)

2. Robichaux-Viehoever, A., Kanter, E., Shappell, H., Billheimer, D., Jones, H., MahadevanJansen, A.: Characterization of Raman spectra measured in vivo for the detection of cervical dysplasia. Applied Spectroscopy 61, 986-993 (2007)

3. Jo, J.A., Fang, Q., Papaioannou, T., Baker, J.D., Dorafshar, A.H., Reil, T., Qiao, J.H., Fishbein, M.C., Freischlag, J.A., Marcu, L.: Laguerre-based method for analysis of timeresolved fluorescence data: application to in-vivo characterization and diagnosis of atherosclerotic lesions. Journal of Biomedical Optics 11 (2006)

4. Noonan, D.P., Elson, D., Mylonas, G., Darzi, A., Yang, G.-Z.: Laser Induced Fluorescence and Reflected White Light Imaging for Robot-Assisted Minimally Invasive Surgery. IEEE Transactions on Biomedical Engineering 56, 889-892 (2009)

5. Ti, Y., Lin, W.-C.: Effects of probe contact pressure on in vivo optical spectroscopy. Optics Express 16, 4250-4262 (2008)

6. Brouwer, I., Ustin, J., Bentley, L., Sherman, A., Dhruv, N., Tendick, F.: Measuring In Vivo Animal Soft Tissue Properties for Haptic Modeling in Surgical Simulation. In: Medicine Meets Virtual Reality, pp. 69-74 (2001)

7. Wagner, C.R., Stylopoulos, N., Howe, R.D.: The Role of Feedback in Surgery: Analysis of Blunt Dissection. In: 10th Symp. on Haptic Interfaces For Virtual Envir. \& Teleoperator Systs., pp. 68-74 (2002)

8. Misra, S., Reed, K.B., Douglas, A.S., Ramesh, K.T., Okamura, A.M.: Needle-Tissue Interaction Forces for Bevel-Tip Steerable Needles. In: IEEE International Conference on Biomedical Robotics and Biomechatronics, pp. 224-231 (2008) 